\author{
Manfred Kohlhase ${ }^{a}$ \\ Ute Wölfle ${ }^{b}$ \\ Christoph M. Schempp ${ }^{b}$
}

\section{Die traditionelle Verwendung von Bitterstoffen}

Schon von Anfang an spielten in der 5000 Jahre alten Ayurvedischen Medizin bitter schmeckende Heilkräuter eine wichtige Rolle. Bitterstoffe reduzieren nach ayurvedischer Auffassung das Fett, reinigen das Blut und tonisieren Muskelgewebe. Auch in der Traditionellen Chinesischen Medizin (TCM) haben Bitterstoffe als fester Bestandteil ausgewogener Ernährungskonzepte eine lange Tradition. Der bittere Geschmack ist in der TCM dem Element Feuer und somit der Hitze, dem Intellekt, dem Handeln, der Freude, dem Lachen und dem Wachstum zugeordnet.

Von dem griechischen Arzt Hippokrates (460-360 v. Chr.) ist überliefert, dass sich unter den von ihm verwendeten und beschriebenen 260 Arzneimitteln 31 Bittermittel befanden. Er verordnete sie als Vorbeugemittel für die unterschiedlichsten Erkrankungen. Später setzte Hildegard von Bingen (1098-1179) sie in ihrer Klostermedizin ein. Das berühmte «Elixir ad longam vitam» des Paracelsus (14931541) soll überwiegend BitterstoffPflanzen enthalten haben, unter anderem Aloe, Galgant und Enzianwurzel. Es wurde im Mittelalter von Apothekern oft in eigener abgewandelter $\mathrm{Zu}$ sammensetzung als Allheilmittel verkauft. Auch das Elixir Proprietatis, be-

aStuttgart, Deutschland; 'borschungszentrum skinitial Klinik für Dermatologie und Venerologie, Universitätsklinikum Freiburg, Freiburg i.Br., Deutschland

\title{
Bitterstoffe als belebende Wirkstoffe für die Haut
}

Bitterstoffe aus verschiedenen Pflanzen spielen seit der Begründung der Ayurvedischen Medizin vor 5000 Jahren bis in die heutige Zeit eine wichtige Rolle als verdauungsanregende und tonisierende Heilmittel. Erst in den letzten Jahren wurden die Bitterstoff-Rezeptoren entschlüsselt und biochemisch charakterisiert. Auch in der Haut wurden Bitterstoff-Rezeptoren nachgewiesen. Bitterstoffe aus dem Enzian und der Weidenrinde binden an die Rezeptoren der Haut; es kommt zu einem Einstrom von Calcium und zur Bildung von Proteinen und Lipiden in den Keratinozyten. Somit beleben Bitterstoffe den Stoffwechsel der Haut und regenerieren die Hautbarriere. Dies kann zum Beispiel für die Behandlung der Neurodermitis genutzt werden.

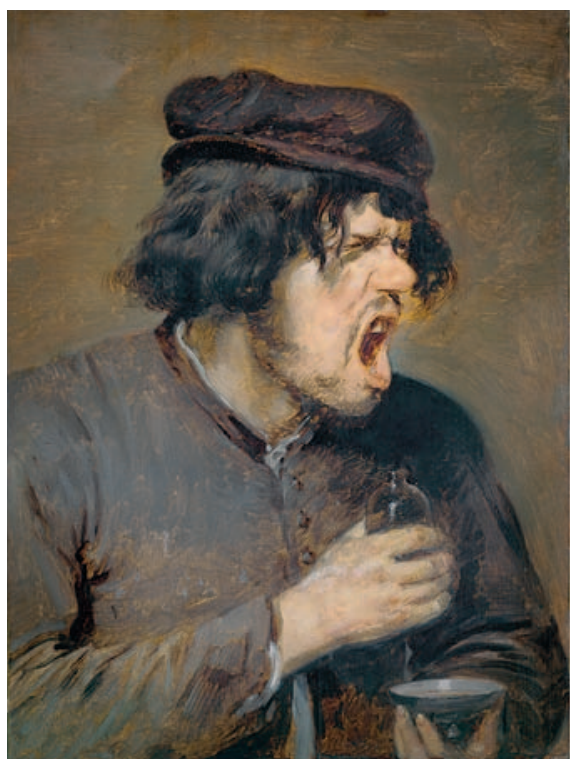

Abb. 1. «Der bittere Trunk» von Adriaen Brouwer (1636).

stehend aus den bitteren Bestandteilen Myrrhe, Aloe und Safran, geht auf Paracelsus zurück. Es diente dann im 17. Jahrhundert dem schwedischen Arzt Dr. Urban Hjärne (1641-1724) als Vorbild für den berühmten Schwedenbitter, dem Maria Treben mit ihrem 1980 veröffentlichten Bestseller «Gesundheit aus der Apotheke Gottes» schliesslich zu grösserer Bekannt- heit verhalf. Der Schwedenbitter enthält unter anderem Aloe, Myrrhe, Sennesblätter, Kampfer, Rhabarberwurzel, Zitwerwurzel und Angelikawurzel. Mit dem bitteren Geschmack von Medizin verbinden allerdings viele Menschen ein unangenehmes Erlebnis. Adriaen Brouwer, der flämische Maler, hat dies um 1636 in seinem Bild «Der bittere Trunk» treffend zum Ausdruck gebracht (Abb. 1).

Die medizinische Bedeutung der Bitterstoffe ging dann bis in das 20 . Jahrhundert immer weiter zurück. Sie wurden nur noch als Aperitif und Magenbitter zur Appetitanregung verwendet und aus dem Gemüse immer mehr herausgezüchtet, bis dann um die Jahrtausendwende die BitterstoffRezeptoren entdeckt wurden und eine Renaissance der bitteren Lebensmittel und Heilpflanzen einsetzte.

\section{Bitterstoffe aus pharmazeutischer Sicht}

Bitterstoff-Heilpflanzen werden als sogenannte "Amara» nach ihren Inhaltsstoffen oder sensorischen Qualitäten eingeteilt [1]. Heute sind ca. 250

\section{KARGER}

(C) 2017 S. Karger GmbH, Freiburg

Fax +497614520714 


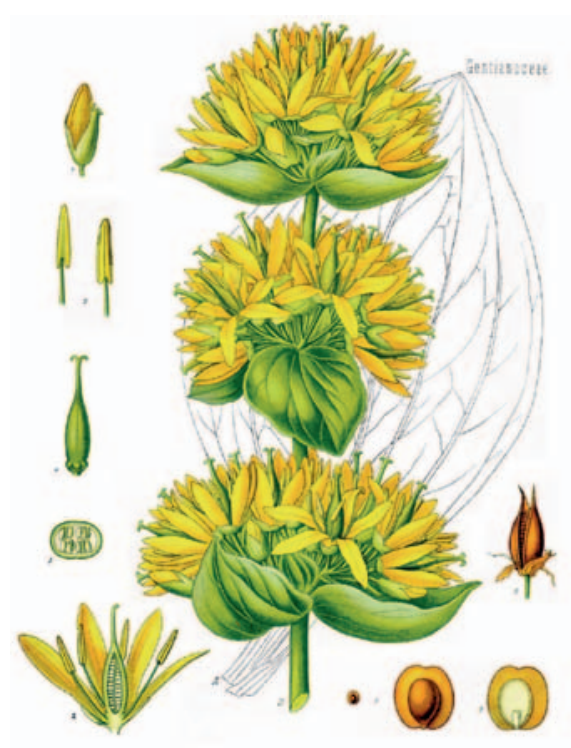

Abb. 2. Gelber Enzian (Gentiana lutea) aus Köhlers Lexikon der Medizinalpflanzen (1897).

bitter schmeckende medizinisch verwendete Pflanzen bekannt. Als eine der bittersten Pflanzen gilt Gentiana lutea (Abb. 2), der Gelbe Enzian, dessen Wurzeln unter anderem das Amarogentin $(0,02-0,04 \%)$ enthalten, das bis $\mathrm{zu}$ einer Verdünnung von 1: 58000000 noch als bitter wahrgenommen wird (Abb. 3).

Weitere natürliche Bitterstoffe sind die giftigen Cucurbitacine (Bitterstoffe in Gurken- und Kürbisgewächsen wie der Zaunrübe), die Alkaloide der Chinarinde, Sesquiterpenlactone wie das Cynaropikrin (Hauptbitterstoff der Artischocke) oder das Cnicin (ein Bitterstoff des Benediktenkrautes), die Simarubalide (wie das Quassin und andere Bitterstoffe der Quassia amara) und verschiedene Iridoide (Loganin, Gentiopicrosid), die in den Bitterkleegewächsen und den Enziangewächsen vorkommen.

Bitterstoff-Pflanzen und ihre Extrakte gelten als appetitanregend, sekretionsfördernd und motilitätssteigernd auf die Darmperistaltik. Deshalb eignen sie sich zur Behandlung von Appetitlosigkeit, dyspeptischen Beschwerden bzw. Magen-Darm-Beschwerden und Störungen des Gallenflusses [1]. In der Phytotherapie werden den Bittermitteln zusätzlich auch

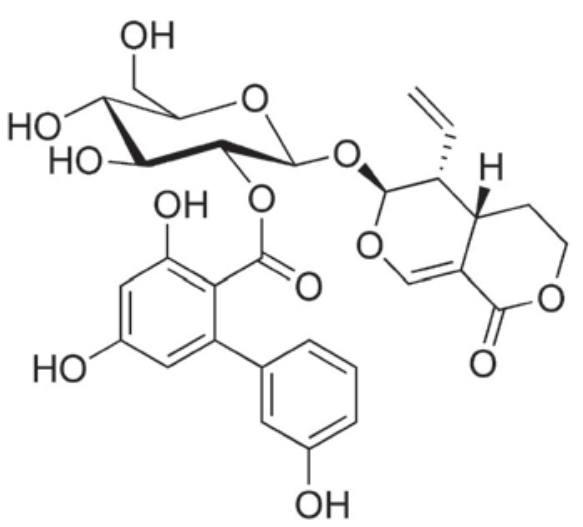

Abb. 3. Strukturformel von Amarogentin (Grafik: Forschungszentrum skinitial).

antidepressive und adaptogene Eigenschaften zugeschrieben, denn sie werden mit Erfolg auch bei Müdigkeit, Erschöpfung, Stress oder allgemein bei somatoformen Störungen eingesetzt [1].

\section{Die Entdeckung der Bitterstoff-Rezeptoren}

So lang auch die Tradition der Verwendung von Bitterstoffen als appetitanregende und tonisierende Heilmittel ist, so war doch bis Ende des letzten Jahrhunderts kaum etwas über die Physiologie des Schmeckens bekannt. Man wusste zwar, dass sich das Schmecken auf der Zunge und im Mund konzentriert. Doch erst 1992 gelang es, ein Signaltransduktionsprotein nachzuweisen, das in Geschmackszellen spezifisch exprimiert wird - das Gustducin [2] (Tab. 1). In der renommierten Fachzeitschrift Nature Medicine wurde beschrieben, dass Bitterstoff-Rezeptoren auch in den Bronchien exprimiert werden und bei Aktivierung zu einer Bronchodilatation führen [3]. Seitdem mehren sich die Hinweise, dass Bitterstoff-Rezeptoren nicht nur im oberen Verdauungstrakt, sondern im gesamten Magen-Darm-Trakt und darüber hinaus in nahezu allen anderen extraintestinalen Organen vorhanden sind [4].

\section{Auch die Haut besitzt \\ Bitterstoff-Rezeptoren}

Im Jahr 2015 gelang erstmals der Nachweis von Bitterstoff-Rezeptoren in menschlicher Epidermis [5]. Untersuchungen des Forschungszentrums skinitial an der Universitäts-Hautklinik Freiburg im Breisgau haben gezeigt, dass pflanzliche Bitterstoffe, z.B. Amarogentin aus dem Gelben Enzian und Salicin aus der Weidenrinde, an Bitterstoff-Rezeptoren der Haut binden und einen Calcium-Einstrom induzieren. In der Folge kommt es zur Bildung von Schutzproteinen, die beim Aufbau der Hautbarriere eine wichtige Rolle spielen [5]. Noch unveröffentlichte Untersuchungen derselben Arbeitsgruppe weisen darauf hin, dass Bitterstoffe auch die Synthese von Hautlipiden in Keratinozyten anregen. Diese Hautlipide werden ebenfalls für den Aufbau der Hautbarriere benötigt. Somit können Bitterstoffe bei der Pflege von trockener Haut mit einer geschädigten Barriere von Nutzen sein, z.B. bei Kontaktekzem und Neurodermitis. Insgesamt werden der Stoffwechsel und die Regeneration der Epidermis angeregt. Die in letzter Zeit in den Medien viel gepriesene belebende Wirkung der Bitterstoffe dient somit auch als Jungbrunnen für die Haut.

\section{Die Anwendung von \\ Bitterstoffen an der Haut}

Die äusserliche Anwendung von Bitterstoffen an der Haut war bisher nicht üblich. Da die Bitterstoff-Rezeptoren der Haut erst 2015 entdeckt wurden, steht die Forschung zur Wirkung von Bitterstoffen auf die Haut noch ganz am Anfang.

Eine klinische Studie der Universitäts-Hautklinik mit äusserlich angewendeten Bitterstoffen und Süssholzextrakt zeigte bei Patienten mit leichter Neurodermitis bereits nach einwöchiger Anwendung eine rasche Besserung der Symptome (Seiwerth J, 
Tab. 1. Geschichte der Erforschung des Schmeckens (nach [8])

\begin{tabular}{ll}
\hline 350 v. Chr. & $\begin{array}{l}\text { Aristoteles beschreibt erstmals die Geschmacksrichtungen bitter und süss und ihre } \\
\text { Modifikation durch die Geschmacksrichtungen salzig und sauer }\end{array}$ \\
\hline 1901 & $\begin{array}{l}\text { Hanig beschreibt die unterschiedliche Verteilung der Geschmacksrichtungen auf der } \\
\text { Zunge; diese «Landkarte des Schmeckens» ist heute jedoch nicht mehr gültig }\end{array}$ \\
\hline 1965 & $\begin{array}{l}\text { Farbman beschreibt die Entwicklung der Geschmacksknospen auf den Papillen der } \\
\text { Zunge von Ratten }\end{array}$ \\
\hline $\begin{array}{l}\text { McLaughlin entdeckt ein geschmacksspezifisches G-Protein in den Geschmacksknospen } \\
\text { der Zunge, das Gustducin }\end{array}$ \\
\hline $\begin{array}{l}\text { Chandrashekar entdeckt den ersten Geschmackssensor, die Bitterstoff-Rezeptoren (T2R); } \\
\text { in den folgenden Jahren werden 25 Bitterstoff-Rezeptoren beim Menschen beschrieben }\end{array}$ \\
\hline 2001 & Nelson entdeckt die Süss-Rezeptoren (T1R) \\
\hline 2010 & $\begin{array}{l}\text { Amerikanische Wissenschaftler entdecken Bitterstoff-Rezeptoren auch auf dem } \\
\text { respiratorischen Flimmerepithel und in den Bronchien }\end{array}$ \\
\hline $\begin{array}{l}\text { Das Forschungszentrum skinitial entdeckt Bitterstoff-Rezeptoren in der Haut und } \\
\text { weist deren Aktivierung durch verschiedene natüliche Bitterstoffe nach }\end{array}$ \\
\hline
\end{tabular}

Tasiopoulou G, Hoffmann J, Schempp CM, Wölfle U: Die topische Anwendung von Bitterstoffen und Süssholzextrakt wirkt entzündungshemmend und ist wirksam bei Neurodermitis. Publikation in Vorbereitung).

Bitterstoffe wirken in der Haut nicht nur direkt auf Keratinozyten, sondern haben auch immunmodulierende Wirkungen im Zusammenspiel von Mastzellen mit Keratinozyten [6]. Es konnte z.B. gezeigt werden, dass Amarogentin die Substanz-P-induzierte Produktion des Entzündungs- mediators TNF- $\alpha$ aus humanen Mastzellen hemmt. Ausserdem reduziert Amarogentin in Keratinozyten die TNF- $\alpha$, und Histamin induzierte die IL(Interleukin)-8- und MMP(MatrixMetalloproteinasen)-1-Expression, was die Einwanderung von Entzündungszellen verringert. Diese Wirkung ist vergleichbar mit dem Antihistaminikum Azelastin und könnte auch bei der Neurodermitis zum Tragen kommen.

Innerlich werden Bitterstoffe bei Hautkrankheiten vor allem in der An- throposophischen Medizin angewen$\operatorname{det}[7,8]$. In der Anthroposophischen Medizin hat sich bei der Behandlung der Neurodermitis - ähnlich wie beim Asthma bronchiale und der Pollinosis (Heuschnupfen) - die abwechselnde Gabe von Gerbstoffen (morgens) und Bitterstoffen (abends) bewährt [9]. Es ist durchaus vorstellbar, dass Bitterstoffe auch von innen (über Nahrungsmittel und Arzneimittel) in die Haut gelangen und dort ihre spezifische Wirkung entfalten.

\section{Ausblick}

Die Entdeckung von Bitterstoff-Rezeptoren in fast allen Organsystemen hat zu einer rasanten Entwicklung der Forschung auf diesem Gebiet geführt. Dies wird auch zu einer allgemeinen Aufwertung der Therapie mit Bitterstoffen führen, die in der Phytotherapie und der Anthroposophischen Medizin eine lange Tradition haben. Bitterstoffe scheinen nicht nur im Magen-Darm-Trakt zu wirken, sondern im gesamten Organismus des Menschen. An der Haut wurden bis jetzt stoffwechselanregende, immunmodulierende und regenerationsfördernde Wirkungen nachgewiesen. Es ist daher vorstellbar, dass Bitterstoffe wie Adaptogene ausgleichend und harmonisierend auf die Haut wirken.

\section{Literatur}

1 Saller R, Melzer J, Uehleke B, Rostock M: Phytotherapeutische Bittermittel. Schweiz Zschr Ganzheitsmedizin 2009;21:200-205.

2 Trivedi BP: The finer points of taste. Nature 2012;486:S2-S3.

3 Deshpande DA, Wang WC, McIlmoyle EL, et al: Bitter taste receptors on airway smooth muscle bronchodilate by localized calcium signaling and reverse obstruction. Nat Med 2010; 16:1299-1304.

4 Lu P, Zhang C-H, Lifshitz LM, ZhuGe P: Extraoral bitter taste receptors in health and disease. J Gen Physiol 2017;149:181-197.

5 Wölfle U, Elsholz FA, Kersten A, Haarhaus B, Müller WE, Schempp CM: Expression and functional activity of the bitter taste receptors TAS2R1 and TAS2R38 in human keratinocytes. Skin Pharmacol Physiol 2015;28:137-146.

6 Wölfle U, Haarhaus B, Schempp CM: Amarogentin displays immunomodulatory effects in human mast cells and keratinocytes. Mediators Inflamm 2015;2015:630128.
7 Grah C: Anwendung von Bitterstoffen in der Anthroposophischen Medizin (AM). Merkurstab 2012;65:165-166.

8 Jachens L: Die Behandlung von Hautkrankheiten über die Leber. Merkurstab 2004;57:248259.

9 Soldner G, Stellmann HM: Individuelle Pädiatrie, ed 4. Stuttgart, WVG, 2014, pp 598-600. 